\title{
The Scientific Programme of JET
}

\section{A. Gibson, Abingdon}

(JET Joint Undertaking)

JET is an experiment designed to explore the possibility of utilising the nuclear fusion of light isotopes as a controlled terrestrial energy source. The two main avenues towards controlled fusion are: inertial confinement, which relies on the implosion of small fuel pellets and magnetic confinement, in which the hot reacting material forms a highly conducting plasma confined by a magnetic field. JET ${ }^{1,2}$ ) uses a magnetic confinement system known as the Tokamak, which evolved in the Soviet Union in the late 1950's. The configuration is shown in Fig. 1. The tokamak is the most advanced of the magnetic confinement systems in that it has produced plasma parameters closer to those required for a self sustained reaction than has been possible with any other system. The parameters which characterise the self sustaining condition are the temperature of the plasma ions ( $T_{i}$ - usually expressed in $\mathrm{keV}$ ) and the product of density (number $/ \mathrm{m}^{3}$ ) and energy confinement time $\left(n \tau_{E^{\prime}} \mathrm{m}^{-3} \mathrm{~s}\right)$.

The most accessible fusion reaction (which may be attempted in JET and will be used in the first fusion reactors) is ${ }^{*}$ :

$$
\begin{aligned}
{ }_{1}^{2} \mathrm{D}+{ }_{1}^{3} \mathrm{~T} \rightarrow & { }_{2}^{4} \mathrm{H}_{\mathrm{e}}+\quad{ }_{0}^{1} \mathrm{n} \\
& 3.52 \mathrm{MeV} \quad 14.06 \mathrm{MeV}
\end{aligned}
$$

- In a reactor the required tritium would be produced by neutron capture in a lithium blanket.
The conditions for this reaction to be self sustaining are:

$$
\begin{aligned}
& T_{\mathrm{i}}>10 \mathrm{keV} \\
& n \tau_{E}>(2 \rightarrow 4) \times 10^{20} \mathrm{~m}^{-3} \mathrm{~s}
\end{aligned}
$$

The numerical factor depends upon radial profiles and operating temperature.

The best values for these parameters so far obtained in experimental tokamaks (which operate in hydrogen or deuterium) are $T_{\mathrm{i}} \sim 2 \mathrm{keV}$ with $n \tau_{E} \sim 5 \times 10^{18} \mathrm{~m}^{-3} \mathrm{~s}$; larger $n \tau_{E}$ values $\left(\sim 3 \times 10^{19} \mathrm{~m}^{-3} \mathrm{~s}\right)$ have been obtained at lower $T_{i}(\sim 1 \mathrm{keV})$ and higher temperatures $\sim 6 \mathrm{keV}$ have been obtained with lower $n \tau_{E}\left(\sim 5 \times 10^{11} \mathrm{~m}^{-3} \mathrm{~s}\right)$. Thus if JET is to achieve a self sustaining reaction it will have to improve the combined product $n \tau_{E} T$ by a factor of about 100 over the best obtained so far. Some idea of the rate of progress can be gained by noting that the value of this product in successive tokamak experiments has increased by a factor of $\sim 6000$ between 1965 and the present time, the increase since 1970 being a factor of 500 and since 1975 a factor of 30 .

\section{The JET Device}

The JET device is illustrated in Fig. 2 and its principal parameters are given in Table 1. It consists essentially of a D-shaped vacuum vessel around which are wound the coils of a toroidal field magnet, and a

Fig. 1 - Schematic of the basic tokamak configuration. In such a configuration the field of the plasma current (poloidal field) provides the main confinement of the plasma while the applied toroidal field is necessary to ensure magnetohydrodynamic stability.

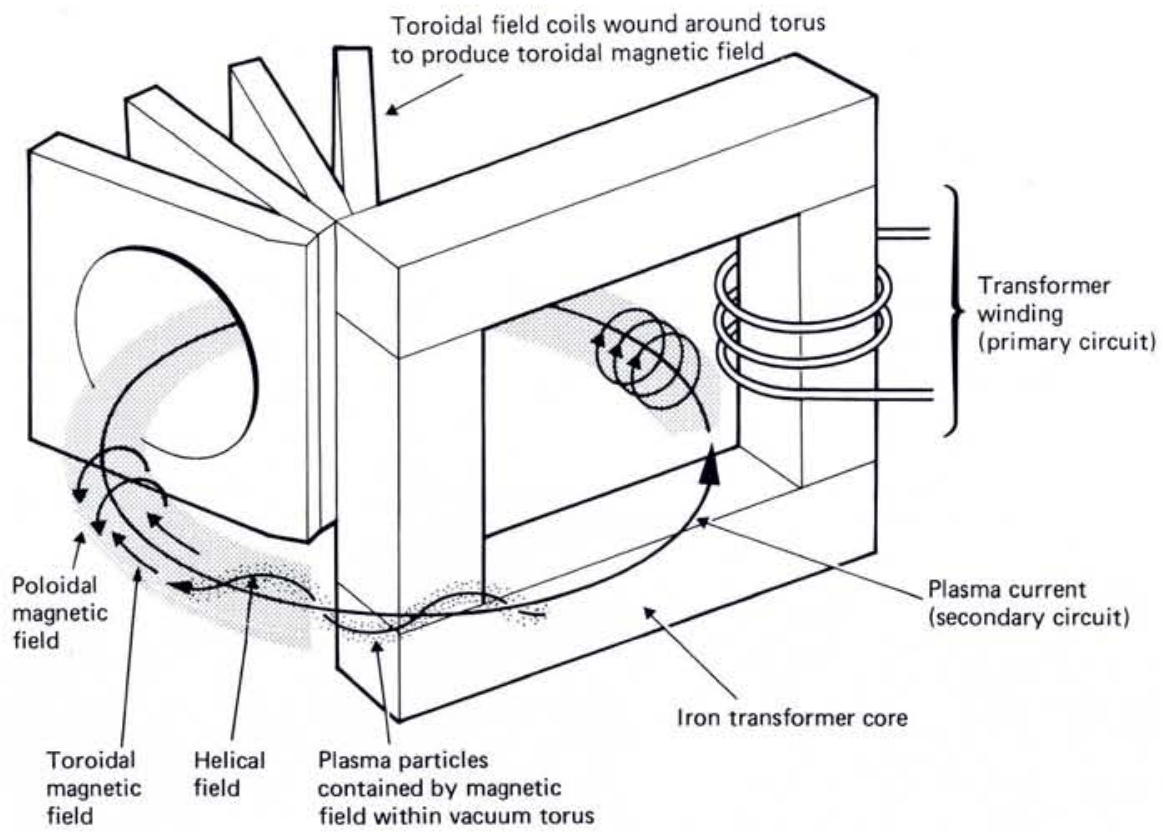

Table 1 - Principal parameters of JET

\begin{tabular}{ll}
\hline Parameter & Value \\
\hline Plasma minor radius (horizontal), $a$ & $1.25 \mathrm{~m}$ \\
Plasma minor radius (vertical), $b$ & $2.10 \mathrm{~m}$ \\
Plasma major radius, $R_{o}$ & $2.96 \mathrm{~m}$ \\
Plasma aspect ratio, $R / a$ & 2.37 \\
Plasma elongation ratio, $b / a$ & 1.68 \\
Flat top pulse length & $10 \mathrm{~s}$ \\
Toroidal magnetic field (plasma centre) & $3.45 \mathrm{~T}$ \\
Plasma current, circular plasma & $3.2 \mathrm{MA}$ \\
\multicolumn{1}{c}{ D-shaped plasma } & $4.8 \mathrm{MA}$ \\
Volt-seconds available & $34 \mathrm{Vs}$ \\
Toroidal field peak power & $380 \mathrm{MW}$ \\
Poloidal field peak power & $300 \mathrm{MW}$ \\
Additional heating power (in plasma) & $25 \mathrm{MW}$ \\
Weight of vacuum vessel & $68 \mathrm{t}$ \\
Weight of toroidal field coils & $380 \mathrm{t}$ \\
Weight of iron core & $2500 \mathrm{t}$ \\
\hline
\end{tabular}

transformer comprising a central laminated iron core with eight symmetrically arranged outer return limbs. The conducting plasma which forms a toroid within the vessel acts as the secondary of the transformer and the current induced in it together with that flowing in the primary transformer coils produce the poloidal field. The primary coils are disposed so that the poloidal field system both drives the plasma current and provides for its equilibrium.

The vacuum chamber (1) is composed of 32 thick double walled sectors joined by bellows and is made from Inconel. This structure is able to resist the forces which arise from atmospheric pressure and from the current induced during the rise and subsequent collapse of the plasma current. At the same time it has a sufficiently large electrical resistance to restrict the flow of toroidal vessel current to an acceptable level. The plasma boundary is defined within the vacuum vessel by a material limiter made from a series of sections forming an axisymmetric ring at large major radius (2). There is also a series of poloida protective shields (3) which prevent the plasma touching the bellows and thin sections of the chamber. The clearance between the largest plasmas envisaged and the vessel itself is $\sim 5 \mathrm{~cm}$ at the small major radius (inside) and $16 \mathrm{~cm}$ at the large major radius. The vacuum vessel is designed to be baked to $500^{\circ} \mathrm{C}$. The vessel is equipped with 8 major horizontal ports and 144 other ports to permit access for the additional heating and for diagnostic measuring systems.

The toroidal field magnet is formed by 32 D-shaped coils (4). The torque which arises where the poloidal field crosses the toroidal field coils is resisted by a mechanical shell (5) which forms an outer torus supporting the toroidal coils.

The poloidal field coils (6) and (7) are connected in parallel in order to simulate a copper shell acting against axisymmetric deformations or displacements of the 


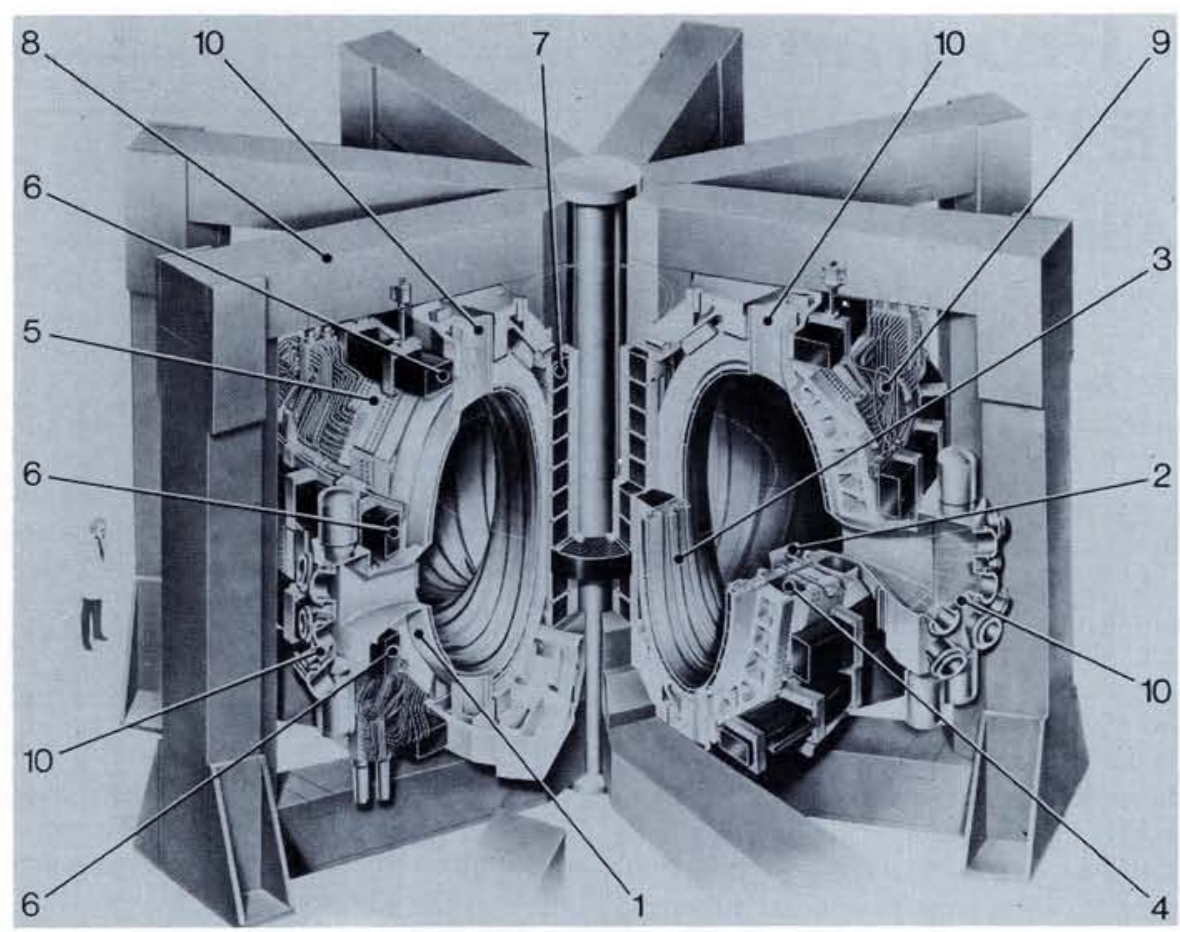

plasma. The poloidal field coils (7) around the central magnetic core create the primary flux, and are split into 10 identical coils which are easier to manufacture than a single coil. In order to obtain the maximum flux, the central section of core is driven far into saturation while the external limbs remain unsaturated.

The toroidal and poloidal field power supplies are a combined static and flywheel system. The two flywheel generators can supply $400 \mathrm{MW}$ and $2.6 \mathrm{GJ}$ each while the static system supplied from the UK $400 \mathrm{kV}$ super grid has a capability of $600 \mathrm{MW}$ for $30 \mathrm{~s}$. The other major subsystems are the Neutral Beam and Radio-frequency (RF) Systems for plasma heating. These systems involve major construction programmes in themselves. The Neutral Beam System designed for $80 \mathrm{kV}$ operation initially and $160 \mathrm{kV}$ operation later, pushes the available technology to the limit. It will be installed in two phases to deliver to the plasma about $10 \mathrm{MW}$ in the principal energy beam component (17 MW including fractional energy components); the corresponding power supply drain will be 96 MW. The RF system will have a supply capable of operating in the range 25 to 55 $\mathrm{MHz}$ and will be installed in phases to deliver $30 \mathrm{MW}$ of RF power to the antenna ( $\geq 15 \mathrm{MW}$ to the plasma interior: $\sim 70$ MW drain).

\section{Project Status}

The project was approved for construction in the summer of 1978. There had been considerable discussion on the site, but eventually a location adjacent to the UK Fusion Laboratory at Culham was chosen. The project is a joint venture of 11 European nations and the European Atomic Energy Community (EURATOM). The ini-

\section{Fig. 3 - The construction site in January 1982.}

Fig. 2 - The JET apparatus:

1. Vacuum vessel (double walled).

2. Material limiter defining the outer plasma edge.

3. Poloidal protective shields to prevent the plasma touching the vessel.

4. Toroidal field magnet of 32 D-shaped coils.

5. Mechanical structure.

6. Outer poloidal field coils.

7. Inner poloidal field coils (primary or magnetising windings).

8. Iron magnetic circuit (core and eight return sections).

9. Water and electrical connections for the toroidal field coils.

10. Vertical and radial ports in the vacuum vessel.

behaviour necessary for the design of a future reactor.

The project development plan foresees four phases in the operation of JET and these are summarised, with the objectives of each phase, in Table 2. More speculatively, planned dates and installed heating power are indicated. The programme as shown is almost entirely paced by the availability of additional heating. In the case of Neutral Injection the dates for higher performance are subject to the outcome of the Neutral Injection development and construction programme; in the case of Radio Frequency heating it depends on the continued success of heating at the lon Cyclotron Resonance Frequency (ICRF). This type of heating has been demonstrated at the few MW level on tokamaks in France, the USA and Japan and appears to be as effective as neutral injection in heating plasma. The overall efficiency may well be several times higher than for Neutral Injection and the design and construction of the RF generators and transmission system are within the experience of the RF industry. However, the design and performance of antennas to launch the power into a plasma with the parameters of JET is an area of uncertainty and there are problems associated with impurity generation during the heating

The following sections describe the aims and programmes of the successive phases of JET operation.

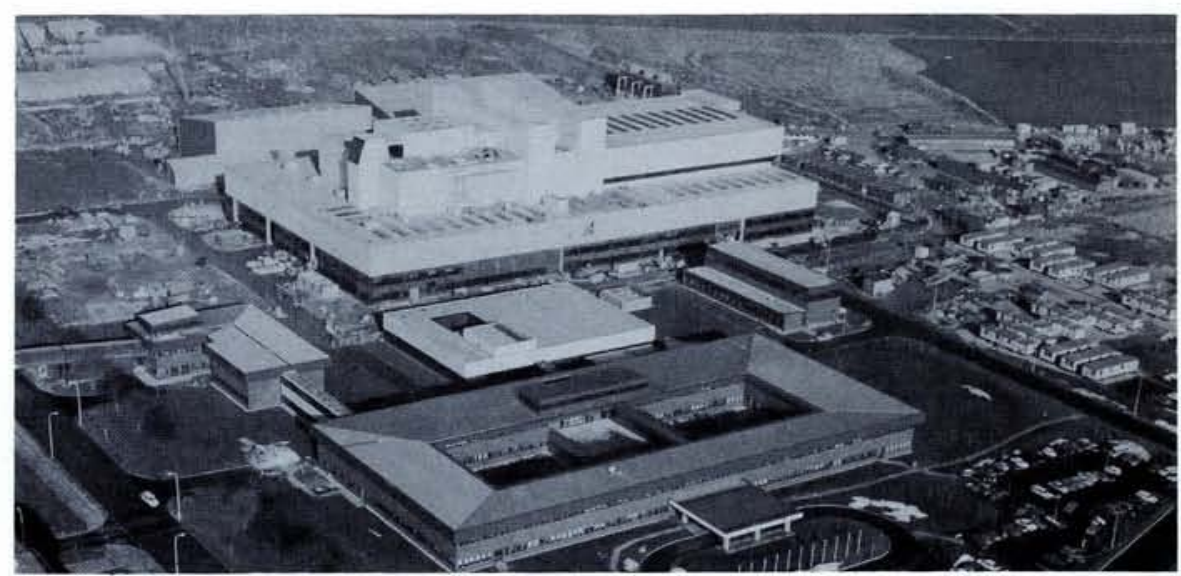




\section{Phase I}

AIM: To learn to operate JET and to establish a clean dense plasma suitable for heating.

This phase will begin in the final stages of machine commissioning with attempts to establish operating regimes at low plasma currents. Attempts will be made to operate with a D-shaped plasma filling the available cross-section. The plasma current will gradually be increased to the limit set by the power supplies and plasma behaviour. A period of at least several months will be necessary to establish reliable operation at currents in the 4 - 5 MA range. Key problems to be solved in this phase are the establishment of clean plasma conditions and the control of elongated plasmas. This ohmic phase will also provide an opportunity to gain experience with the various plasma measurement systems, usually called diagnostics. Interpretation code development will continue, to provide a co-ordinated and integrated measurement and analysis system for the subsequent phases.

Glow discharge and pulse discharge cleaning systems are being constructed for JET. These systems will be evaluated and optimised in this phase. Water-cooled nickel and inertially-cooled carbon clad limiters are being installed in the first instance, and their performance in the unconventional limiter configuration of JET will have to be evaluated. Bolometry, infrared cameras viewing the limiters and plasma surface diagnostics will be important in this phase. The plasma position and shape will be determined by the magnetic diagnostics supplemented by bolometers and Electron Cyclotron Emission (ECE) systems. At the beginning it is probable that only the more basic diagnostics will be available. This will enable some of the gross parameters to be compared with theory: electron temperature as a function of radius, $T_{e}(r)$, electron density, $n_{e}$, and the ion temperature $\left(T_{i}\right)$; estimates of the neutral outflux, temperature of specific wall and limiter locations and some measurement of wall interaction. The remaining diagnostic systems should become available and particularly important will be the spectroscopic diagnostics for impurity studies, X-ray diode arrays for studies of instability behaviour and the improved spatial scan systems. The neutron diagnostics are not likely to be important in this phase but essential components should be present so that their sensitivity to $\mathrm{X}$-rays and response to photo-neutrons can be evaluated.

\section{Phase II}

AIM: to understand and control the plasma and impurity behaviour with significant additional heating, including RF heating.

The power levels shown in Table 2 are power in the principal component for the Neutral Beam and the power which should be delivered to the plasma interior for RF

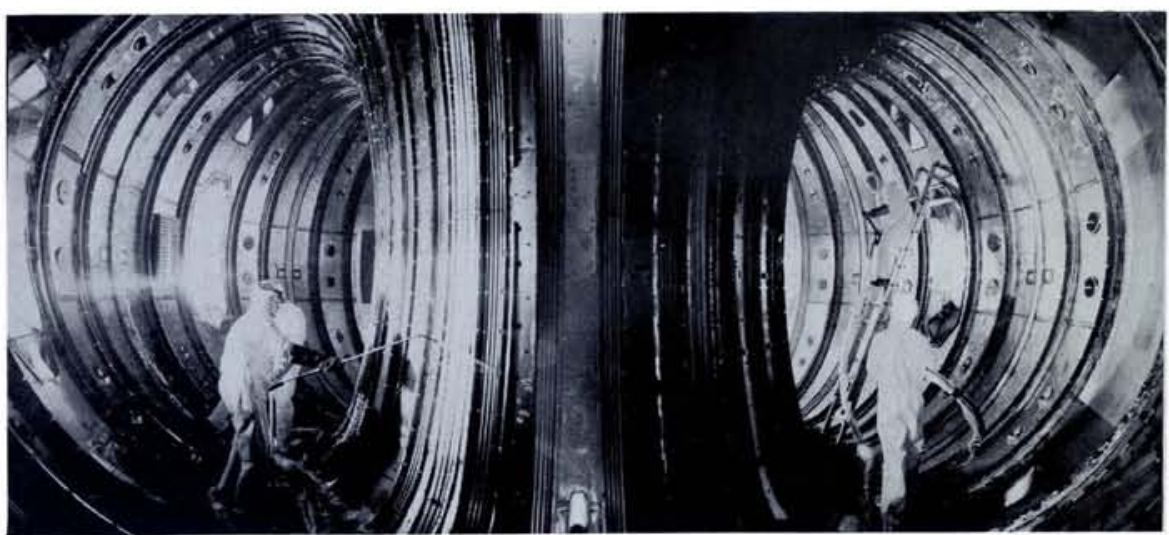

Fig. 4 - Montage showing the tight torus of the vacuum vessel during the final cleaning.

(estimated as $50 \%$ of antenna power). The full set of diagnostic equipment will be required in this phase. The RF work and possibly some of the neutral injection work will require deuterium plasmas and there will be an opportunity to gain experience with the neutron diagnostics and to establish confidence in them as effective measurement systems.

Information will be sought on the buildup of deuterium on the walls which will be important in evaluating tritium inventories for Phase IV. Fuelling methods will be developed to control the $\mathrm{D}-\mathrm{H}$ ratio, again necessary information for Phase IV. Methods will also be developed to measure the D-T ratio in Phase IV (optical and neutron spectra).

An important part of the work in this phase will be to determine the limits on plasma density, to learn how to avoid the major current inhibition (disruption) which sometimes occurs in present tokamaks. Methods of routinely achieving a controlled termination of the current pulse will have to be established. The main thrust of the programme will be to determine and control the limiting loss processes. This work will place particular emphasis on impurity and instability diagnostics.

Detailed comparisons with transport codes will be attempted and interpretive codes will be used to obtain local transport coefficients. Impurity sources and transport behaviour will be investigated. The object will be to establish a good model to predict the behaviour of subsequent phases and to establish the quantity and type of additional heating power that will be required to approach ignition. In this phase it will become clear whether the essential JET configuration and impurity control is sufficient to justify the introduction of tritium in an attempt to establish a self sustaining plasma.

If serious obstacles are encountered in either impurity or instability behaviour the duration of the phase will have to be extended while methods are devised to overcome the difficulties. Should this not be

Fig. 5 - The completed assembly with the installation of bus bars and instrumentation cabling in progress.

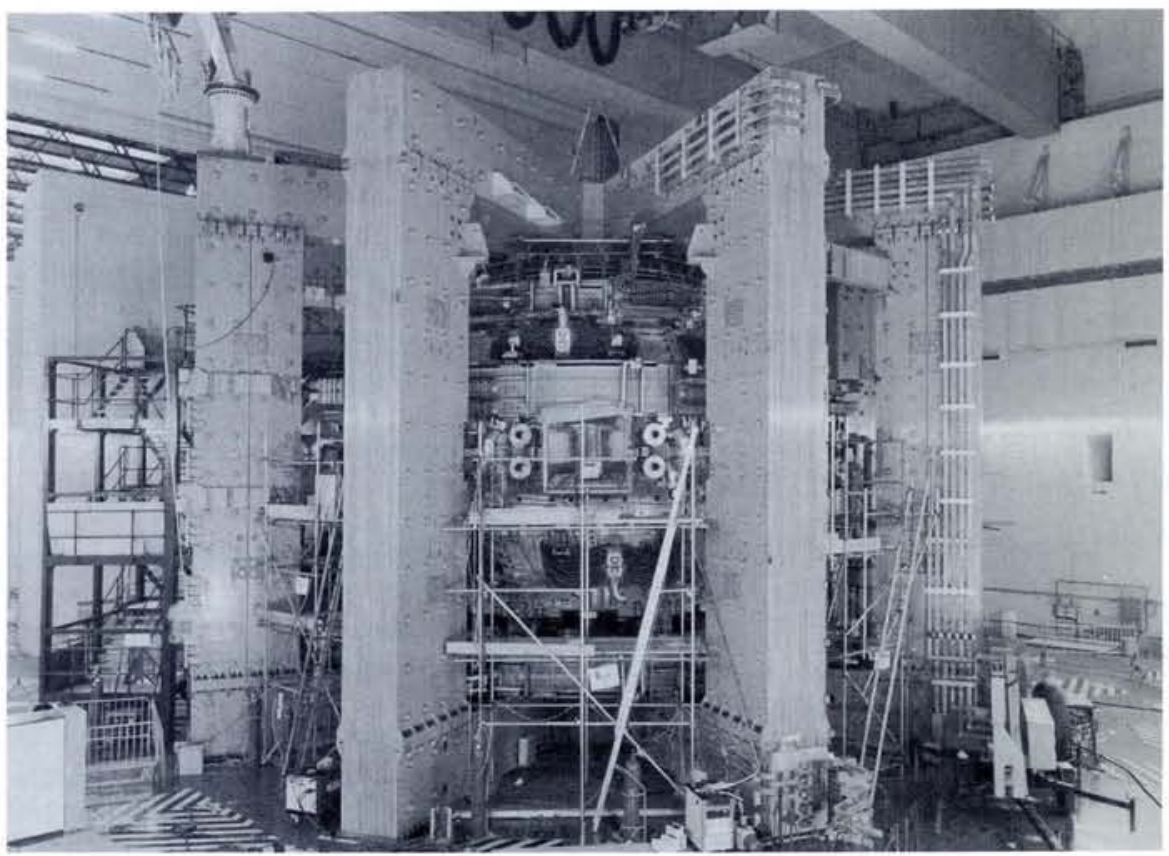


Table 2 - Phases in the Operation of JET Showing the Planning Date and Effective Heating Power (in Addition to Ohmic)

\begin{tabular}{l|l}
\hline Description & Object \\
\hline Phase I mid 1983 & 0
\end{tabular}

Initial Operation and 1. Establish effective

Commissioning with cleaning techniques.

ohmic heating only. 2. Evaluate limiter per-

Mainly $\mathrm{H}_{2}$ operation, formance.

possibly $\mathrm{He}$ and $\mathrm{D}_{2}$. $\quad 3$. Control of $\mathrm{D}$ sha-

ped plasmas.

4. Establish limits on / and $n$.

5. Establish fuelling methods.

6. Compare perfor-

mance $\left(T, \tau_{e}\right)$ with

transport codes.

7. Examine discharge

termination methods.

Phase II A mid $19845 \mathrm{MW}$

Operation with Neutral Injection Heating 5 $\mathrm{MW}$ of $80 \mathrm{keV}(\mathrm{H})$.

1. Develop effective impurity control techniques.

2. Evaluate and optimise heating performance, first with Neutral Injection, then with the addition of ICRH.

3. Establish limits on $n$

Phase II B 1985 $16 \mathrm{MW}$

Additional Heating level: $10 \mathrm{MW} ; 80$ $\operatorname{keV}(\mathrm{H})$ N.I. $+6 \mathrm{MW}$ (effective) ICRH Gases mainly $\mathrm{H}_{2}$, possibly $\mathrm{He}$ and $\mathrm{D}_{2}$ or $\mathrm{H}_{2} / \mathrm{D}_{2}$ mixtures.

\section{Phase III A 1986}

Operation with increased Additional Heating and capability for deuterium injection:

$10 \mathrm{MW}$ : $80 \mathrm{keV}(\mathrm{H})$ or $160 \mathrm{keV}(\mathrm{D})$ of N.I. + $9 \mathrm{MW}$ (effective) ICRH.

\section{Phase III B 1987} $25 \mathrm{MW}$

Operation with maximum planned Additio nal Heating capability: $10 \mathrm{MW}$; $160 \mathrm{keV}(\mathrm{D})$ of N.I. + $15 \mathrm{MW}$ (effective) ICRH.

\begin{tabular}{l|l}
\hline Phase IV 1988 & $25 \mathrm{MW}$ \\
Operation with signifi- & $\begin{array}{l}\text { 1. Study approach to } \\
\text { cant } \alpha \text {-particle heating } \\
\text { ignition. } \\
\text { in deuterium-tritium } \\
\text { gas mixtures. }\end{array}$ \\
$\begin{array}{l}\text { 2. Study plasmas with } \\
\text { profiles determined by } \\
\alpha \text { power deposition } \\
\text { profile. } \\
3 . \text { Examine } \beta \text { limit vio- } \\
\text { lation due to } \alpha \text { hea- } \\
\text { ting. }\end{array}$ \\
\hline
\end{tabular}

possible, the main experimental programme of JET would be diverted to studying the limiting processes and assessing their impact on future reactor plans. In this case Phase II would merge into Phase III with higher heating powers becoming available but mainly being deployed in $\mathrm{H}_{2}$, so as to minimise activation problems. This presents a difficulty for ICRF minority heating where a helium plasma might have to be used to minimise neutron production.

It is during this phase of the experimental programme that various specialised operating scenarios aimed at improving the performance in the face of particular difficulties will be tried. Examples are: the cool plasma mantle scenario aimed at limiting impurity effects by control of the edge region; the expanding plasma mode and its variants to overcome problems of beam penetration and the adiabatic major radius compression mode intended to provide transient high temperatures in the face of short energy replacement times produced by certain loss processes. This last mode would require an extension to the JET power supply. During this phase too it may be necessary to experiment with various limiter, or even wall materials to secure an acceptable impurity content.

By the end of the phase the full range of parameters possible with the available power supplies and heating methods will have been assessed, including performance parameters such as temperature, density and energy replacement time as well as the parameters which characterise the configuration such as plasma current, pressure and elongation.

\section{Phase III}

AIM: to obtain plasma conditions in deuterium which in a D-T mixture would give substantial $\alpha$ particle heating.

At the beginning of Phase III a clear understanding of the plasma behaviour and potential of JET will have been obtained and, on the assumption that new instabilities or impurity sources are not encountered at the higher power levels, reliable predictions of the performance will be available. New problems to be expected have to do with increased wall and limiter loadings. Plasma wall diagnostics will be important as will the $2.4 \mathrm{MeV}$ neutron diagnostics. $14 \mathrm{MeV}$ neutrons will be generated from the tritium reaction product and will afford an opportunity to establish the $14 \mathrm{MeV}$ neutron diagnostics in preparation for Phase IV.

There may well be a need to limit the neutron production during this phase so as to restrict activation levels in the torus structure, although activation levels corresponding to a total neutron production during deuterium operation of $10^{20}$ neutrons could be accepted. This is the number of neutrons to be expected from one full ignition discharge in D-T. At the beginning of the phase the production of this number of neutrons may require some 3000 discharges, but by the time conditions suitable to form a basis for phase IV have been obtained, the $10^{20}$ neutrons should be produced in some 200 discharges.

The intense nuclear radiation levels encountered by the end of this phase will test the radiation tolerance of the diagnostics. Towards the end, the diagnostic configuration will change to that intended for D-T operation, i.e., one or two systems may be upgraded, withdrawn behind the shield wall or abandoned. Many repair and maintenance procedures will require full remote handling, for example: limiter replacement, other in-vessel operations and ion source replacement.

By the end of the phase a clear picture will have been obtained of the conditions which could be produced in D-T mixtures in the absence of $\alpha$ particle heating. The $\alpha$ particle heating to be expected will be accurately predicted and a secure basis will exist for deciding whether the benefit of D-T operation will offset the cost in terms of loss of access to the apparatus and the consequent limitation of the experimental programme.

\section{Phase IV}

AIM: to operate in D-T and move, using as few discharges as possible, to the point where $\alpha$ particle heating dominates the core plasma.

A number of technical changes will be required before Phase IV can begin. The tritium system will have to be brought into operation; various penetrations in the biological shield will have to be filled or provided with increased secondary shields and the remote maintenance systems will have to be fully operational. The diagnostic systems will have already operated in the D-T configuration.

There will exist a good understanding of the plasma behaviour except in so far as it is affected by the fusion power source. The initial discharges will be used to gain control of the tritium fuelling based upon the experience obtained in hydrogen and deuterium operation. Probably the discharge will be initiated at low pressure in deuterium and the pressure increased by tritium gas injection while the neutral injectors inject deuterium. The gas mixture will be controlled in this way to maximise the power (neutron) production. It will be essential at this stage for the diagnostic and interpretation systems to perform with extreme reliability as an integrated whole.

Interest will centre on conditions where $\alpha$ heating is dominant in the central core. An important topic will be the behaviour as theoretical limits to " $\beta$ " are approached, under the influence of fusion heating. $(\beta$ is the ratio of plasma pressure to magnetic pressure and will be an important economic parameter for a reactor.) Possible problems which may be encountered will include in- 
stability and disruption behaviour provoked by the $\alpha$ heat source.

The most important diagnostics in this phase will undoubtedly be the neutron diagnostics, but other systems which are essential in unravelling the energy balance, such as the bolometers will retain a key importance as will diagnostics determining impurity and instability behaviour, such as the spectroscopic and X-ray systems. The wall diagnostics will be required to give information on the plasma wall and radiation damage behaviour in this phase. It would also be useful to have direct information on $\alpha$ particle energy transfer and containment but it is not clear at present that suitable diagnostic techniques can be developed to operate in the intense radiation field. Information on the effectiveness of the $\alpha$ particle heating will however be obtained from the neutron measurements. The various systems giving spatially resolved information on temperature and density profiles will be essential for detailed energy balance analysis.

\section{Time Scales}

The start of Phase I in mid-1983 depends, of course, on the present construction schedule being maintained. The beginning of Phase II, shown as 1984, however, depends on the availability of additional heating and diagnostic equipment which in principle is not linked to the main construction programme. Thus Phase II is subject to delays of its own but is not sequentially dependent on Phase I, which if necessary could be curtailed. It would be possible and indeed preferable to begin directly with additional heating and a full set of diagnostics if these were available. Consequently, it seems reasonable to suppose that some form of Phase II operation will take place during 1984.

The beginning of Phase III operation shown as 1985 depends on the availability of advanced high power heating equipment. This is turn depends on the solution of design and construction problems. The total time allocated for Phases II and III in Table 1 is $3 \frac{1}{2}$ years. In very favourable circumstances and given a full year for Phase I this time might be reduced to $2 \frac{1}{2}$ years. Of course if impurity, instability or reliability problems prove serious in the earlier phases, this date will be delayed and, in the worst case, D-T operation will not be undertaken in JET at all. Nevertheless, the date of 1988 shown in the Table for commencement of D-T operation appears to be reasonable, although it does assume a certain element of good fortune backed by sound planning, timely resource commitment and forceful management.

\section{REFERENCES}

1. Palumbo D., The European Great Projects (EPS) 1979, p. 35

2. Gibson A., "The JET Project", Naturwissenschaften 66 (1978) 481.

\title{
Hydrogen in Intermetallic Compounds
}

\author{
L. Schlapbach, Zurich \\ (Laboratorium für Festkörperphysik, ETH)
}

Intermetallics are single phase stoichiometric compounds of two or more elemental metals. Many of them react readily with gaseous hydrogen and form exothermically, hydrides of convenient stability which can be used for reversible $\mathrm{H}_{2}$ storage. The high density of the stored $\mathrm{H}$, the good kinetics of the $\mathrm{H}$ absorption and desorption, as well as safety aspects, make $\mathrm{H}_{2}$ storage by intermetallics very attractive and are the main reasons for the rapid growth of applied research in this field over the last ten years. The related phase transitions, structure transformations, variations of the electronic and magnetic properties of bulk and surface, phonon properties of the metal lattice and of the interstitial $\mathrm{H}$ and $\mathrm{H}$ diffusion represent a broad and exciting field for basic research in solid state and material science.

The actual research is concentrated on compounds of the following four groups: - $\mathrm{AB}_{2}$ compounds (e.g. $\mathrm{ZrMn}_{2}, \mathrm{TiMn}_{2-\mathrm{x}^{\prime}}$ so called Laves phases)

- $A B_{5}$ compounds le.g. LaNi $i_{5}$, which is the "drosophila" of the $\mathrm{H}_{2}$ storage compounds)

- FeTi based compounds

- $\mathrm{Mg}$ based compounds (e.g. $\mathrm{Mg}_{2} \mathrm{Ni}$, $\mathrm{La}_{2} \mathrm{Mg}_{17}$, alloys of $\mathrm{Mg}$ )

As is evident from these examples, at least one of the components is a hydride former ( $\mathrm{Zr}$, Rare Earth, Ti, Mg, Pd,...).

Whereas many physical and chemical properties of hydrides of intermetallic compounds (ternary hydrides) are comparable with those of hydrides of elemental metals (binary hydrides), there are also some pronounced differences:

- intermetallics are, by far, more reactive to $\mathrm{H}_{2}$ and less sensitive to impurities in the gas;

- the brittle intermetallics disintegrate into powder;

- $\mathrm{H}$-bonding is weaker and can be adjusted by substitutions or through offstoichiometric mixtures.

\section{Phase Diagrams}

The $\mathrm{H}$ absorption behaviour can be described in terms of pressure-composition-isotherms (Fig. 1). The intermetallics are able to dissolve some atomic percent $\mathrm{H}$ in the $\alpha$ phase, but then as the $\mathrm{H}$ concentration is increased, the $\mathrm{H}-\mathrm{H}$ interaction becomes locally important and nucleation and growth of the hydride phase $(\beta)$ start. Whilst the two phases $(\alpha+\beta)$ co-exist, the isotherms show a plateau, the length of which determines how much $\mathrm{H}_{2}$ can be stored reversibly with small pressure variations. In the pure $\beta$ phase, the $\mathrm{H}_{2}$ pressure rises steeply with the concentration. At higher $\mathrm{H}_{2}$ pressure further plateaus and further hydride phases $(\gamma, \sigma, \ldots)$ may be found. The two phase region ends in a critical point $T_{c}$.

For $\mathrm{LaNi}_{5}$ the first plateau is at 1.6 bar at room temperature and the $\alpha$ and $\beta$ phase correspond to $\mathrm{LaNi}_{5} \mathrm{H}_{0.4}$ and $\mathrm{LaNi}_{5} \mathrm{H}_{6}$, respectively. A further plateau exists above $1 \mathrm{kbar}$ corresponding to the formation of $\mathrm{LaNi}_{5} \mathrm{H}_{9}$.

\section{Crystal Structure}

From neutron and $\mathrm{X}$-ray diffraction as well as from the shape of the pressure composition isotherms, it is known that the dissolved $\mathrm{H}$ goes on tetrahedral or octahedral interstitial sites of the metal lattice. Lattice expansions of $\approx 20 \mathrm{vol} \%$ between the hydride phase $(\beta)$ and the solid solution phase $(\alpha)$ are normal. As a result, the brittle intermetallics disintegrate into powder when they go for the first time through the two phase region. After a few absorption / desorption cycles powder grains smaller than $10 \mu \mathrm{m}$ with many cracks and defects are created (Fig. 2), yielding a large specific surface of $\approx 1 \mathrm{~m}^{2} / \mathrm{g}$.

The formation of a high density of lattice defects with the $\mathrm{H}$ absorption leads to an anisotropic broadening of the diffraction lines which makes the unambiguous indexing of the powder pattern difficult, particularly in the case of $\mathrm{LaNi}_{5} \mathrm{H}_{6}$, for which two crystallographically different structure models ( $\mathrm{P} 31 \mathrm{~m}$ and $\mathrm{P} 6 / \mathrm{mm}$ ) have been proposed.

At room temperature, the $\mathrm{H}$ atoms are randomly distributed among the available

Fig. 1 - Pressure-composition isotherms and phase diagram of a metal-hydrogen system.

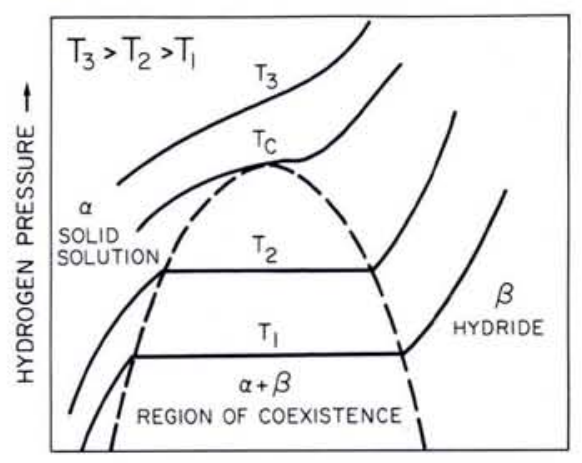

HYDROGEN CONCENTRATION $\rightarrow$ 